Acta Crystallographica Section D

Biological

Crystallography

ISSN 0907-4449

Kiran Kulkarni, Ziguo Zhang, Leifu Chang, Jing Yang, Paula C. A. da Fonseca and David Barford*

Division of Structural Biology, Institute of Cancer Research, Chester Beatty Laboratories, 237 Fulham Road, London SW3 6JB, England

Correspondence e-mail: david.barford@icr.ac.uk

\title{
Building a pseudo-atomic model of the anaphase-promoting complex
}

The anaphase-promoting complex $(\mathrm{APC} / \mathrm{C})$ is a large $\mathrm{E} 3$ ubiquitin ligase that regulates progression through specific stages of the cell cycle by coordinating the ubiquitindependent degradation of cell-cycle regulatory proteins. Depending on the species, the active form of the APC/C consists of 14-15 different proteins that assemble into a 20 -subunit complex with a mass of approximately 1.3 MDa. A hybrid approach of single-particle electron microscopy and protein crystallography of individual APC/C subunits has been applied to generate pseudo-atomic models of various functional states of the complex. Three approaches for assigning regions of the EM-derived $\mathrm{APC} / \mathrm{C}$ density map to specific APC/C subunits are described. This information was used to dock atomic models of APC/C subunits, determined either by protein crystallography or homology modelling, to specific regions of the APC/C EM map, allowing the generation of a pseudo-atomic model corresponding to $80 \%$ of the entire complex.

In this article, we describe an example of molecular replacement in which we use atomic models to interpret electron-density maps determined using single-particle electron-microscopy data. The method of combining highresolution structural information from crystallography with lower resolution structures obtained through electron microscopy is referred to as the hybrid method. We will briefly explain how the APC/C functions to regulate the cell cycle before describing our approach to determining a pseudoatomic structure of the complex.

The APC/C is a large multi-subunit complex that functions as an E3 ubiquitin ligase to regulate progression through the cell cycle by mediating the destruction of cell-cycle proteins via the ubiquitin proteasome pathway (Peters, 2006; Sullivan \& Morgan, 2007; Barford, 2011; Pines, 2011). The APC/C controls the destruction of proteins such as securin, cyclins and the Aurora and Polo mitotic kinases, the activities of which inhibit progression through distinct cell-cycle phases. The destruction of securin at metaphase triggers sister chromatid segregation and the onset of anaphase at mitosis. The APC/C interacts with its substrates through destruction motifs, predominantly the D box and KEN box, mediated by coactivator subunits that bind to the core $\mathrm{APC} / \mathrm{C}$ during mitosis and G1.

The complex functions of the $\mathrm{APC} / \mathrm{C}$ are reflected in its complex organization and size. Human $\mathrm{APC} / \mathrm{C}$ is an assembly
Received 28 February 2013

Accepted 4 July 2013 
of 15 different proteins including the coactivator subunits (Table 1). Their masses range from around $200 \mathrm{kDa}$ for Apc1 to $9 \mathrm{kDa}$ for the smallest subunit Cdc26. We know from native mass spectrometry and crystallography that many subunits are present as two copies per complex, so that the overall mass

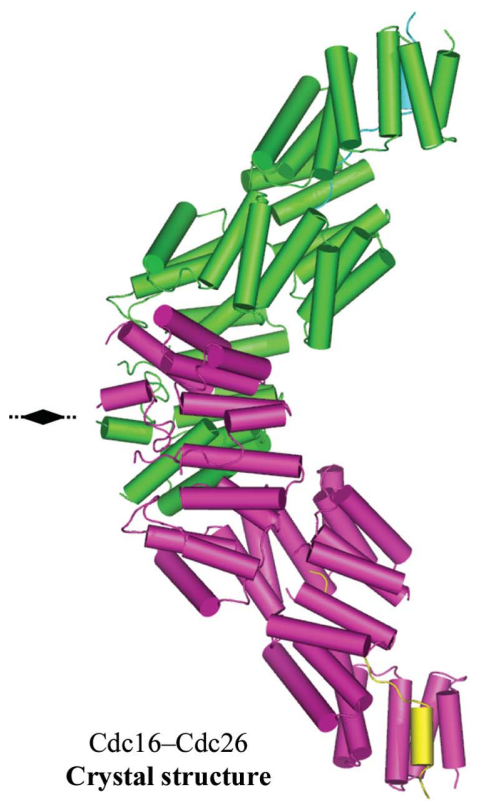

(a)

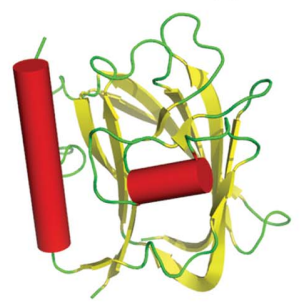

Apc 10

Crystal structure

(c)

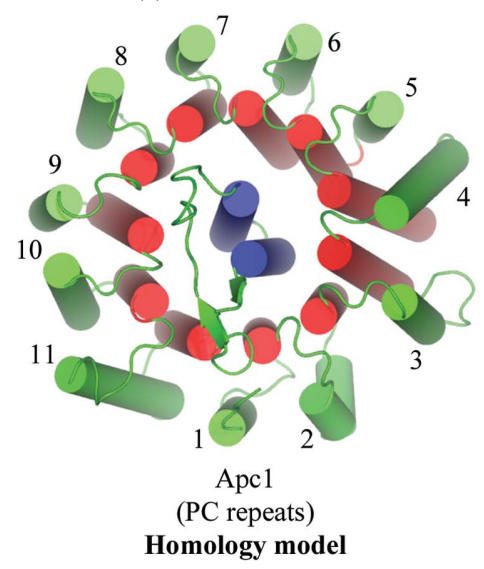

(e)

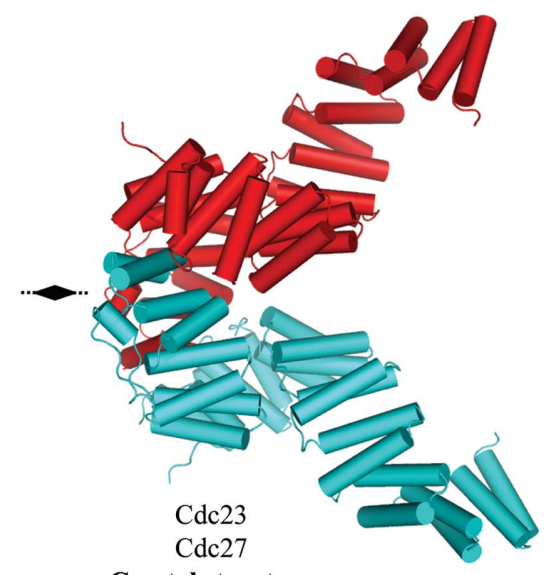

Crystal structure is around 1.3 MDa, depending on the species. Knowing the subunit stoichiometry of a complex is important for accurate fitting of atomic models into the EM structure. Saccharomyces cerevisiae and Schizosaccharomyces pombe APC/C differ from metazoan APC/C by lacking the tetratricopeptide (TPR) subunit Apc7.

One striking feature of the $\mathrm{APC} / \mathrm{C}$ is that only four proteins are involved in directly recognizing target proteins and in catalyzing the assembly of a polyubiquitin chain onto the substrate. All other subunits, which account for $80 \%$ of the mass of the $\mathrm{APC} / \mathrm{C}$, provide scaffolding functions to organize the catalytic and substrate-recognition subunits. Another feature of the APC/C is that many of the scaffolding proteins are structurally related. In human APC/C there are four TPR subunits that have very simple architectures based on multiple contiguous copies of a 34-amino-acid sequence motif called the tetratricopeptide (TPR) motif. The smaller TPR accessory subunits stabilize the larger TPR subunits and also mediate inter-TPR interactions. In isolation, these TPR accessory subunits are disordered and they only assume a defined conformation when associated with the TPR subunits.

Our research has been aimed at obtaining atomic structures of individual APC/C subunits and to define how these subunits are organized within the whole complex as a means to understand how the APC/C recognizes its substrates and catalyses the assembly of polyubiquitin chains. To apply the hybrid approach to generate pseudoatomic structures of the $\mathrm{APC} / \mathrm{C}$ we have determined atomic models of most of the large APC/C subunits through a combination of protein crystallography and homology modelling. We now have atomic models for most of the large APC/C subunits except for Apc4 and the N-terminus of Apc1. We also lack structural information on some of the smaller APC/C subunits (Table 1).

An example of one of the canonical TPR subunits, Cdc16 associated with its accessory subunit Cdc26, is shown in Fig. 1(a) (Zhang, Kulkarni et al., 2010). Two Cdc16 molecules self-associate to form V-shaped homodimers. Each Cdc16 subunit consists of 14 contiguous TPR motifs that create an array of 28

Figure 1

Gallery of APC/C subunit structures. (a) Cdc16-Cdc26 complex (PDB entry 2xpi; Zhang, Kulkarni et al., 2010). (b) Cdc23 (PDB entry 3zn3; Zhang et al., 2013) and Cdc27 (PDB entry 3kae; Zhang, Roe et al., 2010) have similar structures to Cdc16. (c) Apc10 (PDB entry 1gqp; Au et al., 2002). (d) Model of the Apc2-Apc11 complex modelled on Cul1-Rbx1 of the SCF complex (PDB entry 1ldk; Zheng et al., 2002). (e) PC domain of Apc1 modelled on Rpn2 (PDB entry 4ady; He et al., 2012). (f) WD40 domain of Cdc20 (PDB entry 4aez; Chao et al., 2012).
(WD40 repeats)

al structure

$(f)$ 
Table 1

Subunits of the APC/C.

ND, not defined.

\begin{tabular}{|c|c|c|c|c|c|c|c|c|}
\hline Homo sapiens & $\begin{array}{l}\text { Molecular } \\
\text { mass (kDa) }\end{array}$ & $N$ & S. cerevisiae & S. pombe & Structural motif & Function & $\begin{array}{l}\text { Model } \\
\text { determination }\end{array}$ & $\begin{array}{l}\text { Mapping } \\
\text { approach }\end{array}$ \\
\hline \multicolumn{9}{|l|}{ Core subunits } \\
\hline Apc1 & 216.5 & 1 & Apc1 & Cut4 & $\alpha$-Helices and PC repeats & Scaffolding subunit & PC domain homology & Docking/elimination \\
\hline Apc2 & 93.8 & 1 & Apc2 & Apc2 & Cullin homology & Catalytic/Apc11 binding & Homology & Docking \\
\hline Apc3 & 91.9 & 2 & Cdc27 & Nuc2 & TPR & Cdh1/Apc10 binding & X-ray/homology & Subunit deletion \\
\hline Apc4 & 92.1 & 1 & Apc4 & Lid1 & Unknown & Scaffolding subunit & ND & $\begin{array}{l}\text { Fab-Apc4 EM } \\
\text { negative stain }\end{array}$ \\
\hline Apc5 & 85.1 & 1 & Apc5 & Apc5 & Extended TPR & Scaffolding subunit & Homology & Elimination \\
\hline Apc6 & 71.7 & 2 & Cdc16 & Cut9 & TPR & Scaffolding subunit & X-ray & Subunit deletion \\
\hline Apc7 & 66.9 & 2 & - & - & TPR & Cdh1/Apc10 binding & X-ray/homology & Subunit deletion \\
\hline Apc8 & 68.8 & 2 & Cdc23 & Cut23 & TPR & Scaffolding subunit & X-ray/homology & Subunit deletion \\
\hline- & - & - & Apc9 & - & Unknown & Cdc27 stabilizing & & \\
\hline Apc10 & 21.2 & 1 & Apc10/Doc1 & Apc10 & Doc homology/IR motif & Substrate recognition & X-ray & Subunit deletion \\
\hline Apc11 & 9.8 & 1 & Apc11 & Apc11 & RING H2 & Catalytic/E2 binding & Homology & ND \\
\hline Apc13 & 8.5 & 1 & Apc13/Swm1 & Apc13 & Unknown & Cdc23 stabilizing & ND & ND \\
\hline- & - & - & - & Apc14 & Unknown & Unknown & & \\
\hline Apc15 & 14.3 & 1 & Mnd2 & Apc15 & Unknown & MCC/Ama1 regulator & ND & ND \\
\hline Apc16 & 11.7 & $1-2$ & - & - & Unknown & TPR stabilizing & ND & ND \\
\hline Cdc26 & 9.8 & 2 & Cdc26 & Hen1 & Extended chain $/ \alpha$-helix & Cdc16 stabilizing & X-ray & Subunit deletion \\
\hline \multicolumn{9}{|l|}{ Coactivators } \\
\hline $\mathrm{Cdc} 20$ & 54.7 & 1 & Cdc20 & Slp1 & WD40/IR motif & Substrate recognition & & Subunit deletion \\
\hline Cdh1 & 55.2 & 1 & Cdh1 & Ste9 & WD40/IR motif & Substrate recognition & & Subunit deletion \\
\hline- & - & - & Ama1 & Mfr1 & WD40/IR motif & Substrate recognition & & ND \\
\hline
\end{tabular}

antiparallel $\alpha$-helices generating a right-handed TPR superhelix with two complete turns of superhelix. The other TPR subunits (Cdc23 and Cdc27) have similar architectures to Cdc16 (Fig. 1b), as does Apc7 (not shown; Zhang, Roe et al., 2010; Zhang et al., 2013). The crystal structure of Apc10, a subunit that functions as the D-box co-receptor in cooperation with an activator subunit (either $\mathrm{Cdc} 20$ or $\mathrm{Cdh} 1$ ), has been determined ( $\mathrm{Au}$ et al., 2002; Wendt et al., 2001; Fig. 1c). We have derived homology models for some APC/C subunits that we have not yet crystallized. The APC/C catalytic centre, formed by the cullin subunit Apc2 associated with the RINGdomain subunit Apc11, was modelled based on the SCF structure (Fig. 1d; Zheng et al., 2002). The PC (proteasome-

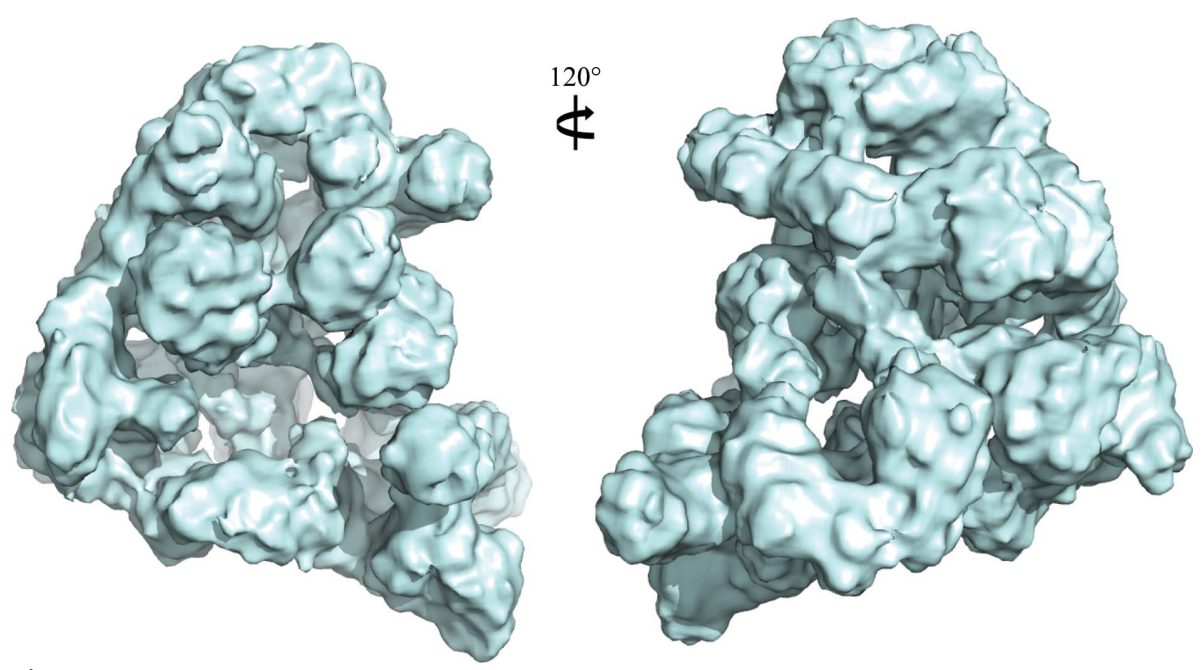

Figure 2

Two views of the cryo-EM reconstruction of S. cerevisiae APC/C in complex with coactivator Cdh1 and a high-affinity D-box peptide (da Fonseca et al., 2011). cyclosome) domain of Apc1 was modelled on the PC domain of Rpn2, a proteosomal subunit, the structure of which we recently determined (Fig. 1e; He et al., 2012). Interestingly, in addition to the TPR subunits, the PC domain of Apc1, and the coactivator subunits, are based on multiple repeat motifs. Coactivators are seven-bladed $\beta$-propeller proteins as shown for the structures of Cdc20 (Fig. 1f) and Cdh1 (Chao et al., 2012; He et al., 2013).

We used single-particle electron microscopy to determine three-dimensional reconstructions of the whole APC/C. Two views of a three-dimensional reconstruction of the $S$. cerevisiae APC/C based on cryo-EM data calculated to $11 \AA$ resolution are shown in Fig. 2 (da Fonseca et al., 2011). Overall, the APC/C adopts a triangular shape measuring $250 \AA$ in the longest dimension. The molecule has an open latticelike appearance and there are clear indications of rod-like and curved tubular densities, some of which correspond to the TPR superhelices and Nterminal cullin repeats of Apc2. To interpret this EM structure, and to generate a pseudo-atomic model of the $\mathrm{APC} / \mathrm{C}$, we have to locate the position of atomic models of $\mathrm{APC} / \mathrm{C}$ subunits within the molecular envelope. We applied three approaches to assign regions of the electron-density map to specific subunits or domains. One approach to segment the APC/C EM density is to generate subcomplexes of the $\mathrm{APC} / \mathrm{C}$ missing defined subunits. By determining the three-dimensional 
structures of these subcomplexes and comparing them with the holo $\mathrm{APC} / \mathrm{C}$, the difference densities can be assigned to the missing subunits (da Fonseca et al., 2011; Schreiber et al., 2011).

To generate the holo $\mathrm{APC} / \mathrm{C}$ and define subcomplexes, we overexpressed recombinant $\mathrm{APC} / \mathrm{C}$ using the MultiBac insectcell expression system (Schreiber et al., 2011). To reconstitute the budding-yeast $\mathrm{APC} / \mathrm{C}$ of 13 proteins, we generated two baculoviruses for co-infection in insect cells. This system works well and we can purify $2-3 \mathrm{mg}$ of $\mathrm{APC} / \mathrm{C}$ per 51 of insect cells, an increase in yield of some 500-fold compared with endogenous APC/C (Schreiber et al., 2011). We observe a
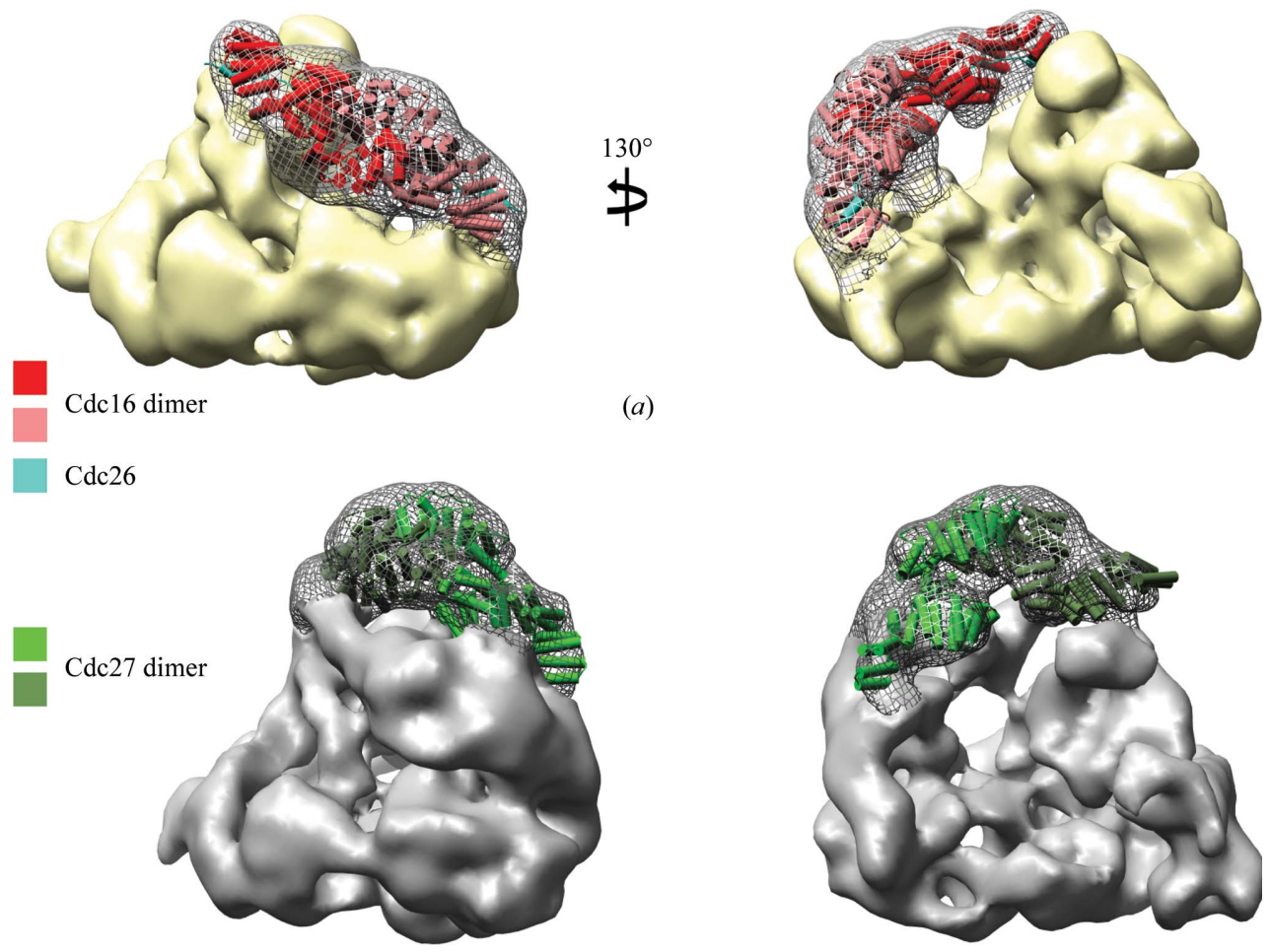

(a)

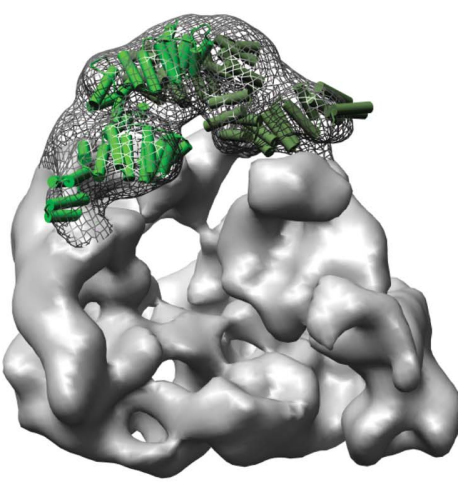

(b)

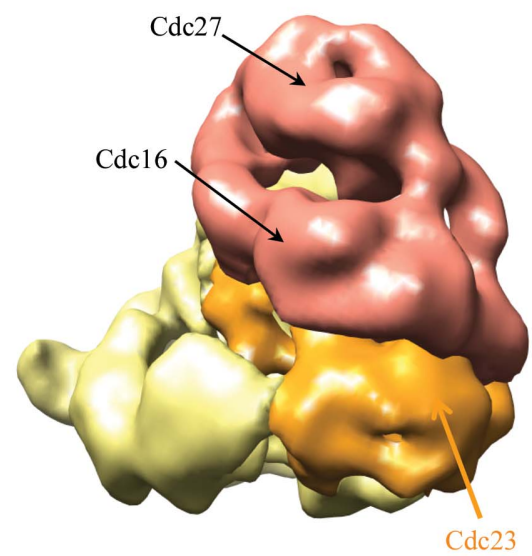

(c)

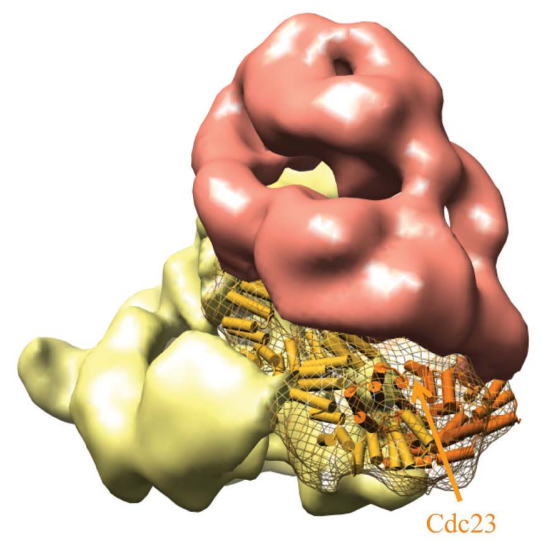

Figure 3

Examples of the subunit-deletion approach to define the location and structure of APC/C subunits. (a) Two views showing the Cdc16 difference density in mesh with Cdc16 coordinates superimposed. (b) Two views showing the $\operatorname{Cdc} 27$ difference density in mesh with $\mathrm{Cdc} 27$ coordinates superimposed. (c) Overlapping densities from two APC/C subcomplexes that share Cdc23 in common defines Cdc23 (orange). Cdc23 coordinates are shown in the right panel (Schreiber et al., 2011). TPR6 corresponds to the red plus orange volume, whereas SC8 corresponds to the yellow plus orange volume. direct correspondence between endogenous and recombinant $\mathrm{APC} / \mathrm{C}$ when the $\mathrm{APC} / \mathrm{C}$ is assessed using silver-stained SDSPAGE. The recombinant APC/C is active as an E3 ubiquitin ligase dependent on the coactivator, and D-box and KEN-box destruction motifs within cyclin. Thus, recombinant APC/C recapitulates the activity of endogenous $\mathrm{APC} / \mathrm{C}$.

We determined three-dimensional reconstructions of the recombinant holo APC/C and a variety of subcomplexes using negative-stain electron microscopy. For example, the subcomplex TPR6 comprises the TPR subunits Cdc16, Cdc23 and Cdc27 together with the TPR accessory subunits, whereas the remaining subunits, including the substrate-recognition and catalytic subunits, assemble into a subcomplex termed SC8 that is dependent on Cdc23 for its stable assembly. Both TPR6 and SC8 match their corresponding densities within the holo $\mathrm{APC} / \mathrm{C}$, showing that they adopt stable autonomous structures.

The first approach to dock atomic models of $\mathrm{APC} / \mathrm{C}$ subunits into the $\mathrm{APC} / \mathrm{C} \mathrm{EM}$ structure was to define difference densities by selective subunit deletions (da Fonseca et al., 2011; Schreiber et al., 2011). In this method, we compare the structures of pairs of APC/C subcomplexes that differ in their composition by a specific subunit. This is illustrated in Fig. 3(a) for Cdc16, in which we compare two APC/C subcomplexes, of which only one incorporates Cdc16. The Cdc16 difference density defines both the position of Cdc16 within the molecular envelope and also the overall shape and molecular boundaries of Cdc16. The difference density has an elongated V-shaped structure with twofold symmetry that exactly matches the atomic model of the Cdc16 homodimer (Zhang, Kulkarni et al., 2010). We could apply a similar approach to $\mathrm{Cdc} 27$ by comparing the holo $\mathrm{APC} / \mathrm{C}$ with APC/C lacking the $\mathrm{Cdc} 27$ subunit. The Cdc27 difference density, at the top of the TPR lobe, has a twofold-symmetrical structure that matches the twofold symmetry of the Cdc27 homodimer atomic model (Fig. 3b).

We applied the subunitdeletion approach to five APC/C 
subunits (Table 1). A disadvantage of the method is that it is restricted to cases in which the resultant subcomplexes are large enough and stable enough to be analysed by negativestain electron microscopy. Some subunits play critical scaffolding functions and their deletion prevents the assembly of usably sized subcomplexes. For example, deletion of $\mathrm{Cdc} 23$ results in a failure to generate $\mathrm{APC} / \mathrm{C}$ subcomplexes of reasonable size. However, we could define the position and molecular boundaries of $\mathrm{Cdc} 23$ by superimposing the subcomplexes TPR6 and SC8 onto the intact holo APC/C structure. TPR6 and SC8 share $\mathrm{Cdc} 23$ in common, and therefore their overlapping densities can be assigned to $\mathrm{Cdc} 23$. The Cdc23-assigned density (Fig. 3c) has an almost identical structure to that assigned for $\mathrm{Cdc} 27$, related by an approximate twofold rotation about Cdc16. This is consistent with Cdc23 and Cdc27 being paralogues.

The second approach to map atomic models is to dock directly into EM maps by visual inspection. This method is possible when regions of the EM maps display strong structural features characteristic of the atomic model. This procedure becomes more reliable when the majority of the EM map has been assigned through empirical approaches and the docking can be guided by additional data such as antibody labelling or protein-protein interaction data. For example, the cullin subunit Apc2 has a rod-like $\mathrm{N}$-terminus and a globular C-terminus that interacts with Apc10 (Fig. 1d). A rod-like density feature within the SC8-defined density that incorporates Apc2 closely matched the atomic model of Apc2. Docking Apc2 into this density positions the C-terminus of Apc2 adjacent to Apc10, which we previously docked using the subunit-deletion approach (Fig. 4; da Fonseca et al., 2011; Schreiber et al., 2011).

The third approach to assigning EM densities to subunits applies when there are no existing atomic models and the subunit-deletion approach is not possible. For example, there are no crystal structures or homology models for Apc4; however, having defined the position of all other large $\mathrm{APC} / \mathrm{C}$ subunits we could define the electron density corresponding to Apc 4 by a process of elimination. Apc 4 is an $80 \mathrm{kDa}$ protein; just a little too small to analyse by negative-stain electron microscopy, which is restricted to proteins larger than $100 \mathrm{kDa}$. To aid structural analysis of Apc4 by negativestain electron microscopy, we generated monoclonal antibodies to Apc4, and the Fab-Apc4 complex is now of sufficient size to analyse by EM. Fig. 5(a) shows a gallery of two-dimensional class averages of the Apc4-Fab complex. The Fab is well resolved whereas Apc4 is less well defined overall, indicating that the Fab interacts with a flexible epitope on Apc4. Particular two-dimensional class averages show well defined two-dimensional projections in which structural details are clear. These averages resemble the electron density extracted from the holo APC/C cryo-EM map potentially corresponding to Apc4 (Fig. $5 b$ ). The approach of generating Fab-protein complexes was adopted by Wu et al. (2012) to enable single-particle cryo-EM studies of small proteins (i.e. $100 \mathrm{kDa}$ in size).

By combining these docking results, we generated a partial pseudo-atomic model of the APC/C. The model, based on the recombinant $S$. pombe APC/C cryo-EM structure, which is still being refined and is at about $12 \AA$ resolution (Fig. 6). Here, we have docked the atomic models of $\mathrm{APC} / \mathrm{C}$ subunits into the density and their positions have been refined using a rigid-body approach by means of $U R O$ (Navaza $e t$ al., 2002; rigid-body fitting methods have been reviewed by Wriggers \&
Figure 4

Identification of $\mathrm{APC} / \mathrm{C}$ subunits by visual inspection. In $(a),(b)$ and $(d)$ rod-like density defines Apc2 (da Fonseca et al., 2011). 


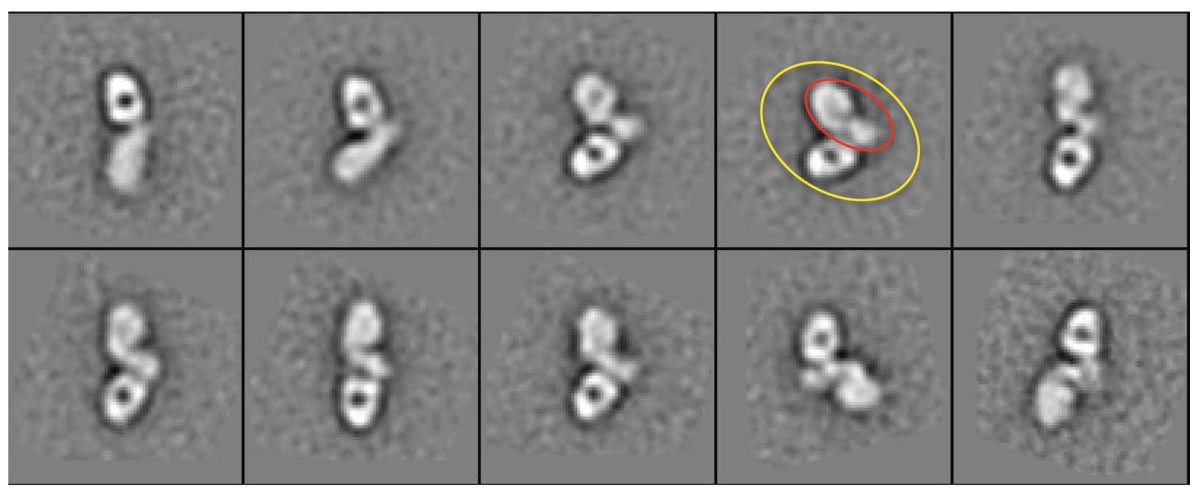

$(a)$

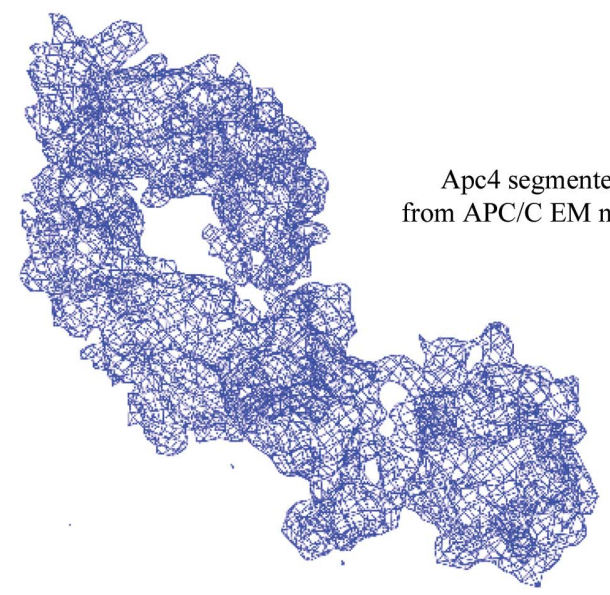

(b)

Figure 5

Apc4 definition. (a) Representative two-dimensional class averages from negative-stain EM micrographs of an Apc4-Fab complex. In one representative class, the Apc4-Fab complex is circled in yellow. Apc4 is circled in red. (b) Density map corresponding to Apc4 and extracted from S. pombe APC/C.

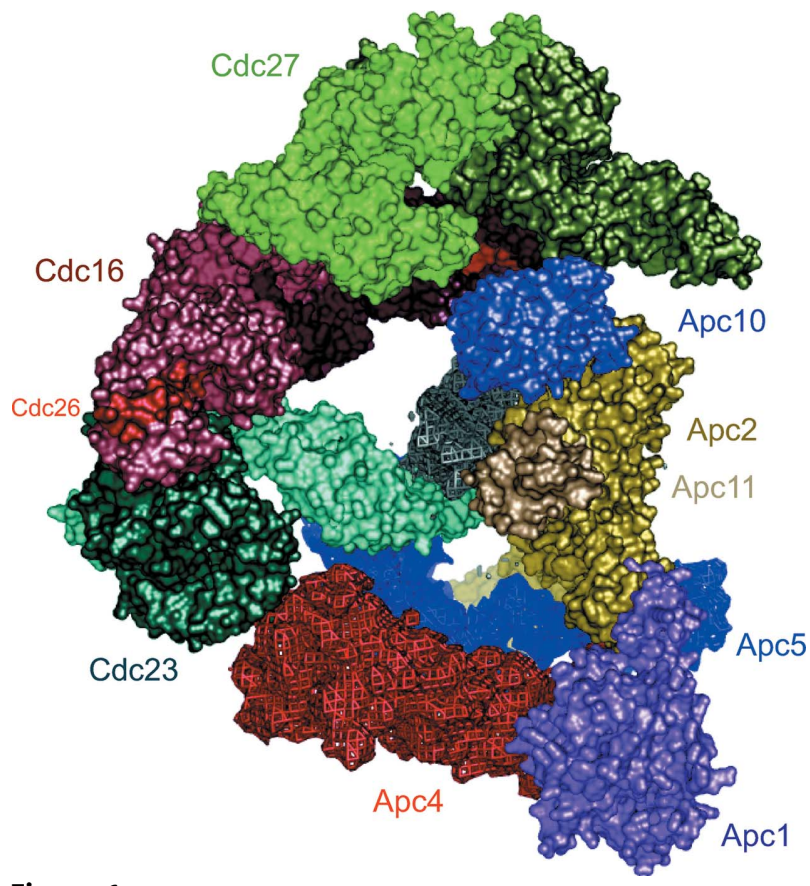

Figure 6

Pseudo-atomic model of the $S$. pombe APC/C. Fitted subunits (Apc2, Apc11, Apc10, Cdc16, Cdc23, Cdc27 and Cdc26) are shown with molecular surfaces. Density assigned to Apc1, Apc4 and Apc5 is shown.
Chacón, 2001). Flexible fitting approaches allow modelling of protein conformational changes and structural differences between the atomic model and the actual structure of the subunit within the context of the multi-subunit assembly (recently reviewed by Trabuco et al., 2009). Such approaches have been implemented in Situs (Wriggers \& Chacón, 2001), MDFF (which employs molecular-dynamics simulations; Trabuco et al., 2008) and DEN, which uses a deformable elastic network model with restraints imposed by the EM map (Schröder et al., 2007, 2010; Brunger et al., 2012). DiMaio et al. (2009) reported the refinement of protein structures into low-resolution density maps using Rosetta, achieving near-atomic resolution starting with EM density maps at 4-6 $\AA$ resolution. Other flexible fitting methods include those reported by Topf et al. (2008) that employ Monte Carlo search, conjugate-gradient minimization and simulated-annealing molecular-dynamics approaches. A method for crossvalidation in cryo-EM-based modelling has recently been proposed (Falkner \& Schröder, 2013).

In the instance of the $\mathrm{APC} / \mathrm{C}$, over $80 \%$ of residues were fitted into the EM map. The coordinates fit well into the EM map. Fig. 6 shows that three canonical TPR subunits, Cdc27, Cdc16 and Cdc23, which are homodimers and structurally related, stack in parallel on one side of the complex. Together they generate a quasi-twofold-symmetrical structure. The N-terminal cullin repeats of Apc2 fit into a long rod-like density placing the C-terminal domain and associated RING subunit Apc11 in close proximity to the subunit-recognition module of Apc10 and coactivator. The subunits for which we have not fitted atomic models are Apc4, Apc5 and the $\mathrm{N}$-terminus of Apc1, and their densities are shown in blue, red and purple, respectively (Fig. 6).

In the future we need to complete the model by determining the crystal structures of Apc4 and the N-terminus of Apc1, and we are aiming to extend the resolution of the EM maps to at least allow the definition of secondary-structural elements. Ideally, we would like to achieve a near-atomic resolution of $<3.5 \AA$. Although this has been achieved for viral structures with high symmetry (60-fold; Zhang, Jin et al., 2010), it is not clear whether this is possible with asymmetric structures, although a recent $4.5 \AA$ resolution structure of the Escherichia coli ribosome indicates that cryo-EM techniques provide the potential to determine near-atomic resolution structures of asymmetric particles (Bai et al., 2012). This paper and other recent studies (Li et al., 2013; Campbell et al., 2012) take 


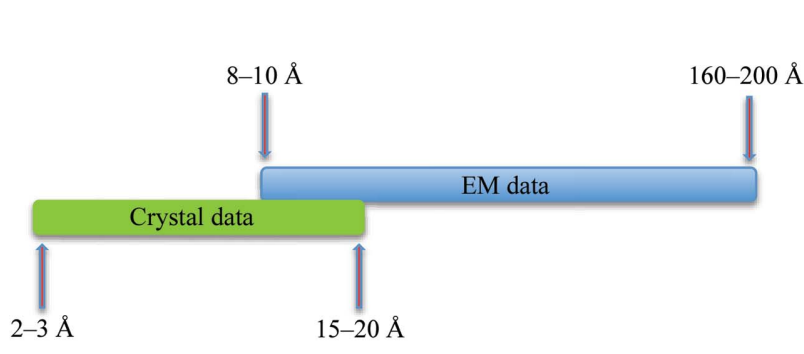

(a)

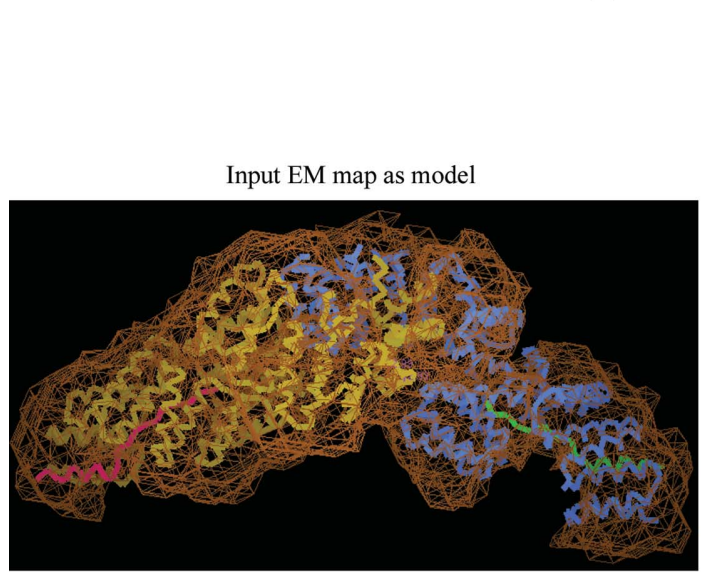

(b)

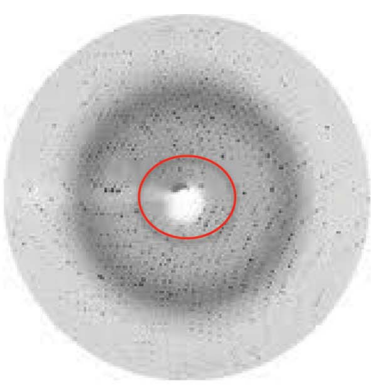

Protocol

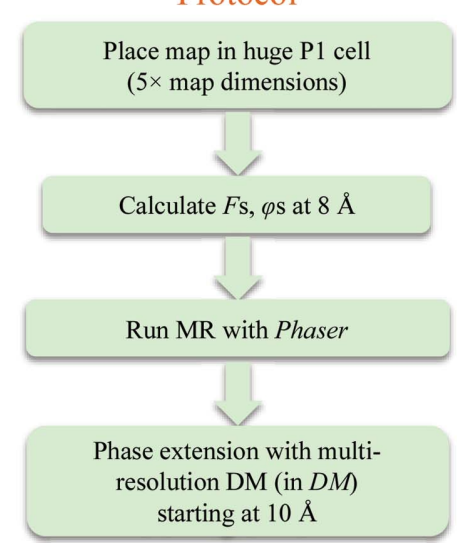

(c)

Figure 7

MR structure solution with an EM map as a model. (a) Schematic of the overlap of EM and X-ray data. (b) Cdc16-Cdc26 assigned density with coordinates. (c) Protocol for using an EM map for crystallographic molecular replacement.
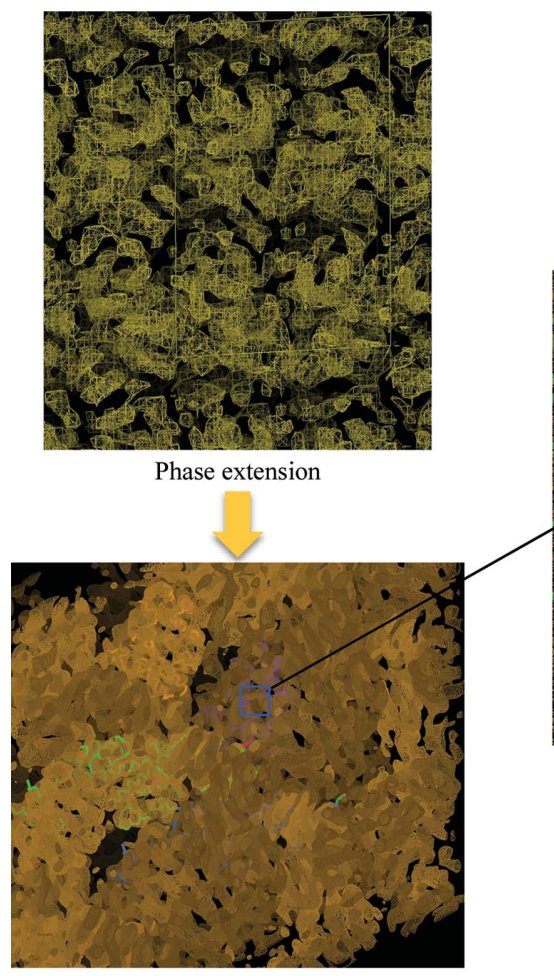

(a)

Figure 8

Cdc16-Cdc26 structure solution with an EM map as a model. (a) The initial map calculated to $10 \AA$ resolution was extended to $5 \AA$ A. (b) The final map shows clear indications of $\alpha$-helices. advantage of the high signal to noise achieved by direct electron detectors. Direct electron detectors also allow 'movie' processing of frames to correct for beam-induced particle motion and also ameliorate loss of resolution owing to radiation-induced sample damage. In addition to improvements in detectors, microscope technology and software [for example, RELION, which implements a Bayesian approach to cryo-EM reconstructions (Scheres, 2012a,b)], biochemical approaches to rigidify multi-subunit complexes will also be important.

As well as using atomic models mainly derived from crystallography to interpret EM-derived electron-density maps, it is also possible to use EM maps to obtain phase information for crystal structure determination. This approach was used by Steitz and coworkers to phase crystals of the large ribosomal subunit (Ban et al., 1998). We tested whether we could use the Cdc16-Cdc26assigned density of our EM map of S. pombe APC/C to phase Cdc16-Cdc26 crystals which we had previously determined using SAD methods (Zhang, Kulkarni et al., 2010). Although there is little resolution overlap between the crystallographic data and the EM data since typical EM data sets range from 8 or 10 to $200 \AA$ resolution, whereas crystallographic data range from 2-3 to $15-20 \AA$ resolution, this approach worked well (Fig. 7a). To test whether the Cdc16-assigned density could be used as a search model in molecular replacement, we placed the EM map corresponding to $\mathrm{Cdc} 16-\mathrm{Cdc} 26$ into a large $P 1$ cell five times the map dimensions to calculate the structure factors (Fig. 7c). The larger size of the cell was to ensure that cross-Patterson vectors calculated for the model, for this particular unit cell, are larger than the radius of integration. Placing the model in a large $P 1$ cell would also improve the signal-to-noise ratio in molecular replacement. Before calculating structure factors, the EM map was not subject to scaling corrections. For molecular-replacement calculations, a resolution cutoff of 4-30 A was applied to the diffraction data. Phases obtained from molecular replacement using 
Phaser (McCoy et al., 2007) were extended from 10 to $5 \AA$ resolution, allowing clear definition of $\alpha$-helices in the crystal structure (Fig. 8). This result validated the quality of the EMderived density maps. We are keen to apply this technique to provide phase information to determine the Apc4 crystal structure.

In summary, we can dock models into EM maps through a difference density approach. The advantage of this method is that is provides empirical constraints to docking and provides information on both the molecular boundaries and position of subunits. A limitation of this approach is that it is dependent on the ability to generate stable subcomplexes. The second approach is to dock by visual inspection, and a third approach, when atomic models are not available, is to use negative-stain EM of individual subunits, possibly aided by generation of subunit-Fab complexes. Generation of a pseudo-atomic model requires atomic models of individual subunits via crystallography or NMR or from structural homologues, and initial docking can be refined by rigid-body refinement and flexible fitting using programs such as URO (Navaza et al., 2002) and MDFF (Trabuco et al., 2008, 2009). Ultimately, if the resolution is sufficient, it is possible to determine structures ab initio using EM data.

The work was funded by a CR-UK programme grant to DB.

\section{References}

Au, S. W. N., Leng, X., Harper, J. W. \& Barford, D. (2002). J. Mol. Biol. 316, 955-968.

Bai, X.-C., Martin, T. G., Scheres, S. H. W. \& Dietz, H. (2012). Proc. Natl Acad. Sci. USA, 109, 20012-20017.

Ban, N., Freeborn, B., Nissen, P., Penczek, P., Grassucci, R. A., Sweet, R., Frank, J., Moore, P. B. \& Steitz, T. A. (1998). Cell, 93, 1105-1115.

Barford, D. (2011). Philos. Trans. R. Soc. Lond. B Biol. Sci. 366, 3605-3624.

Brunger, A. T., Adams, P. D., Fromme, P., Fromme, R., Levitt, M. \& Schröder, G. F. (2012). Structure, 20, 957-966.

Campbell, M. G., Cheng, A., Brilot, A. F., Moeller, A., Lyumkis, D., Veesler, D., Pan, J., Harrison, S. C., Potter, C. S., Carragher, B. \& Grigorieff, N. (2012). Structure, 20, 1823-1828.

Chao, W. C. H., Kulkarni, K., Zhang, Z., Kong, E. H. \& Barford, D. (2012). Nature (London), 484, 208-213.

DiMaio, F., Tyka, M. D., Baker, M. L., Chiu, W. \& Baker, D. (2009). J. Mol. Biol. 392, 181-190.

Falkner, B. \& Schröder, G. F. (2013). Proc. Natl Acad. Sci. USA, 110, 8930-8935.
Fonseca, P. C. A. da, Kong, E. H., Zhang, Z., Schreiber, A., Williams, M. A., Morris, E. P. \& Barford, D. (2011). Nature (London), 470, 274-278.

He, J., Chao, W. C. H., Zhang, Z., Yang, J., Cronin, N. \& Barford, D. (2013). Mol. Cell, 50, 649-660.

He, J., Kulkarni, K., da Fonseca, P. C. A., Krutauz, D., Glickman, M. H., Barford, D. \& Morris, E. P. (2012). Structure, 20, 513521.

Li, X., Mooney, P., Zheng, S., Booth, C. R., Braunfeld, M. B., Gubbens, S., Agard, D. A. \& Cheng, Y. (2013). Nature Methods, 10, 584-590.

McCoy, A. J., Grosse-Kunstleve, R. W., Adams, P. D., Winn, M. D., Storoni, L. C. \& Read, R. J. (2007). J. Appl. Cryst. 40, 658-674.

Navaza, J., Lepault, J., Rey, F. A., Álvarez-Rúa, C. \& Borge, J. (2002). Acta Cryst. D58, 1820-1825.

Peters, J. M. (2006). Nature Rev. Mol. Cell Biol. 7, 644-656.

Pines, J. (2011). Nature Rev. Mol. Cell Biol. 12, 427-438.

Scheres, S. H. W. (2012a). J. Mol. Biol. 415, 406-418.

Scheres, S. H. W. (2012b). J. Struct. Biol. 180, 519-530.

Schreiber, A., Stengel, F., Zhang, Z., Enchev, R. I., Kong, E. H., Morris, E. P., Robinson, C. V., da Fonseca, P. C. A. \& Barford, D. (2011). Nature (London), 470, 227-232.

Schröder, G. F., Brunger, A. T. \& Levitt, M. (2007). Structure, 15, 1630-1641.

Schröder, G. F., Levitt, M. \& Brunger, A. T. (2010). Nature (London), 464, 1218-1222.

Sullivan, M. \& Morgan, D. O. (2007). Nature Rev. Mol. Cell Biol. 8, 894-903.

Topf, M., Lasker, K., Webb, B., Wolfson, H., Chiu, W. \& Sali, A. (2008). Structure, 16, 295-307.

Trabuco, L. G., Villa, E., Mitra, K., Frank, J. \& Schulten, K. (2008). Structure, 16, 673-683.

Trabuco, L. G., Villa, E., Schreiner, E., Harrison, C. B. \& Schulten, K. (2009). Methods, 49, 174-180.

Wendt, K. S., Vodermaier, H. C., Jacob, U., Gieffers, C., Gmachl, M., Peters, J.-M., Huber, R. \& Sondermann, P. (2001). Nature Struct. Biol. 8, 784-788.

Wriggers, W. \& Chacón, P. (2001). Structure, 9, 779-788.

Wu, S. et al. (2012). Structure, 20, 582-592.

Zhang, X., Jin, L., Fang, Q., Hui, W. H. \& Zhou, Z. H. (2010). Cell, 141, 472-482.

Zhang, Z., Chang, L., Yang, J., Conin, N., Kulkarni, K. \& Barford, D. (2013). J. Mol. Biol., doi:10.1016/j.jmb.2013.04.004.

Zhang, Z., Kulkarni, K., Hanrahan, S. J., Thompson, A. J. \& Barford, D. (2010). EMBO J. 29, 3733-3744.

Zhang, Z., Roe, S. M., Diogon, M., Kong, E., El Alaoui, H. \& Barford, D. (2010). J. Mol. Biol. 397, 1316-1328.

Zheng, N., Schulman, B. A., Song, L., Miller, J. J., Jeffrey, P. D., Wang, P., Chu, C., Koepp, D. M., Elledge, S. J., Pagano, M., Conaway, R. C., Conaway, J. W., Harper, J. W. \& Pavletich, N. P. (2002). Nature (London), 416, 703-709. 